\title{
ESTUDANDO OS COMPORTAMENTOS ONLINE: PREMISSAS E DESAFIOS NO DESENVOLVIMENTO DE UM PAINEL DE UTILIZADORES DA INTERNET
}

\section{STUDYING ONLINE BEHAVIORS: ASSUMPTIONS AND CHALLENGES IN THE DEVELOPMENT OF A PANEL OF INTERNET USERS}

\author{
Branco Di Fátima \\ Centro de Investigação e Estudos de Sociologia (CIES/ISCTE), Portugal \\ brancodifatima@gmail.com \\ Filipe Montargil \\ Escola Superior de Comunicação Social (ESCS), Portugal \\ fmontargil@escs.ipl.pt \\ Sandra Miranda \\ Escola Superior de Comunicação Social (ESCS), Portugal \\ smiranda@escs.ipl.pt
}

\begin{abstract}
RESUMO: Este artigo apresenta e discute as premissas e desafios na construção de um painel online de utilizadores da Internet, com foco na experiência pioneira do Living Lab on Media Content and Platforms (LLMCP). Ao longo de 2018, o LLMCP desenvolveu uma extensão do Google Chrome capaz de monitorizar, em tempo real, a navegação dos utilizadores da Internet através deste browser - o mais difundido entre os portugueses. Para poder iniciar a recolha de informação empírica, através de um painel online de utilizadores da Internet, o projeto adotou um conjunto de procedimentos teóricos, metodológicos e operativos no desenvolvimento tanto da ferramenta de monitorização quanto na captação de utilizadores disponíveis para a inscrição no painel. A ampla disseminação de tecnologias de informação e comunicação (TIC) tem vindo a modificar a forma como as pessoas vivem em sociedade e como organizam as suas relações sociais. Compreender a sociedade de informação passa pela criação de tecnologias e procedimentos para investigar essas novas formas de organização e estruturação dos comportamentos dos indivíduos. Para além do estudo de caso do LLMCP, este artigo problematiza brevemente o impacto dos métodos assentes na inquirição, como a entrevista e o questionário, na pesquisa dos comportamentos online. Os resultados apontam para duas linhas de raciocínio. Por um lado, para a ideia de que a adoção em massa de TIC tem vindo a contribuir para a criação de novas oportunidades, no domínio dos métodos de investigação em ciências sociais e humanas. Por outro lado, para a ideia de que as abordagens inovadoras necessitam de enfrentar os receios relacionados com a vigilância e o controlo, que afligem as sociedades hiperconectadas.
\end{abstract}

PALAVRAS-CHAVE: comportamentos; monitorização; privacidade; métodos de investigação em ciências sociais; Internet.

ABSTRACT: This paper presents and discusses the assumptions and challenges for developing an online panel of Internet users, focusing on the pioneering experience of the Living Lab on Media Content and Platforms (LLMCP). Throughout 2018, LLMCP 
developed a Google Chrome extension able to monitor, in real-time, the navigation of Internet users through this browser - the most widely used by the Portuguese. In order to initiate the collection of empirical information through an online panel of Internet users, the project adopted a set of theoretical, methodological and operational procedures in the development of both the monitoring tool and to attract participants. The dissemination of information and communication technologies (ICT) has been changing the way people live in society and how they organize their social relationships. Understanding that the information society involves the creation of technologies and procedures to investigate these new forms of organization and structuring of individuals' behaviors. In addition to the LLMCP case study, this article briefly discusses the impact of inquiry methods, such as interview and questionnaire, on online behavioral research. The results point two lines of reasoning. On the one hand, to the idea that mass adoption of ICT has contributed to the creation of new opportunities in the field of research methods in the social and human sciences. On the other hand, innovative approaches still need to overcome surveillance and control fears existing in hyper connected societies.

KEYWORDS: behaviours; monitoring; privacy; social research methods; Internet.

\section{Introdução}

A rápida disseminação das novas tecnologias de informação e comunicação (TIC), desde a década de 1990, tem levado a relevantes mudanças na forma como vivemos em sociedade e como organizamos as nossas relações sociais. Recursos como a Internet, os telefones móveis ou as redes Wi-Fi estão a permitir a criação de estruturas de organização social qualitativamente diferentes dos períodos históricos que os antecederam (GREEN, 2010; CASTELLS, 2013; WEST, 2015). Pelo que as experiências quotidianas indicam, e as investigações parecem corroborar, as alterações podem ser observadas em praticamente todas as dimensões da sociedade. Passam pela forma como os conteúdos noticiosos são produzidos e consumidos (SALAVERRÍA, 2016), pela apreciação de eventos desportivos (HUTCHINS; ROWE, 2012), pelos cuidados com a saúde e iniciativas médicas (ESPANHA, 2016), pelas transações económicas e financeiras (ADAPA, 2011), pela organização de protestos e pela mobilização de cidadãos para atos políticos (CARDOSO; LAPA; DI FÁTIMA, 2016).

Mas de que formas nos apropriamos da Internet? Que práticas são mais recorrentes? A resposta a estas perguntas é habitualmente obtida através da utilização de métodos de inquirição. Em diferentes contextos, nacionais (MARKTEST, 2015; OBERCOM, 2014) e internacionais (IBOPE CONECTA, 2018; PEW CENTER, 2016), pesquisas com base em entrevistas e questionários revelam que a vida online é formada pela combinação de ações informativas, comunicativas e de entretenimento. Pesquisamos informação através do Google, comunicamos com familiares e amigos através do Instagram, do Facebook e do Skype, fazemos compras na Amazon e no eBay, vemos filmes no Netflix.

Contudo, os métodos assentes na inquirição também apresentam um conjunto de limitações que, desde a década de 1930, tem despertado o interesse das ciências sociais (LAPIERE, 1934; ROBERTS, 1985). A própria limitação da memória humana para eventos quotidianos pode influenciar a perceção do que as pessoas consideram fazer e aquilo que 
efetivamente fazem (FODDY, 1996). As fragilidades dos métodos de inquirição são ainda mais relevantes em sociedades informacionais maduras e dinâmicas, como a portuguesa, onde a Internet chega a $75 \%$ da população e o telefone móvel é utilizado por $97 \%$ dos indivíduos (INE, 2017; MARKTEST, 2018).

Este artigo apresenta um estudo de caso dos desafios e premissas para a construção de um painel online de utilizadores da Internet, na região de Lisboa, com base na experiência pioneira do Living Lab on Media Content and Platforms (LLMCP). Um dos objetivos do LLMCP consiste em explorar as relações e lacunas entre práticas e representações pela análise comparada dos métodos de inquirição e da monitorização dos comportamentos na Internet. Perante a crescente penetração e assimilação das TIC, e considerando as limitações metodológicas associadas às técnicas de inquirição, as ciências sociais e humanas encontram na própria tecnologia novas oportunidades para o estudo e para a análise da sociedade contemporânea.

Os resultados preliminares do estudo de caso indicam que a adoção de soluções e abordagens inovadoras implica a necessidade de lidar com os receios relacionados com a vigilância e a invasão de privacidade, decorrentes da utilização de tecnologia, nas nossas sociedades hiperconectadas. Se, por um lado, a adoção de TIC pode contribuir para o desenvolvimento de novas oportunidades, no domínio dos métodos de investigação em ciências sociais e humanas e das respetivas técnicas de recolha de informação empírica, por outro lado torna-se crítico sensibilizar as pessoas para a relevância e segurança destas novas abordagens. Por outras palavras, embora a tecnologia seja relevante, as comunidades humanas constituem o cerne das ciências sociais.

\section{Práticas e representações: limitações da inquirição}

As ciências sociais estudam, de forma sistematizada, a relação entre práticas e representações desde, pelo menos, a década de 1930. O trabalho seminal de LaPiere (1934) explorou a relação entre atitudes sociais e padrões comportamentais, em situações concretas.

De acordo com a análise do autor, os indivíduos podem dar respostas, num inquérito, que não correspondem aos seus próprios comportamentos anteriores. Estas respostas podem inclusivamente, aliás, revelar-se contraditórias com esses mesmos comportamentos. Como LaPiere observa, não existe necessariamente correlação entre discurso e ação, entre a resposta verbalizada pelo inquirido e a realidade que ela simboliza (LAPIERE, 1934, p. 231). LaPiere acompanhou as viagens pelos EUA de um jovem casal de origem chinesa, entre 1930 e 1932 - um período em que a atitude dos norte-americanos relativamente à população chinesa era de "distância social" e de preconceito publicamente assumido.

Este casal viajou pela Costa do Pacífico, por diversas ocasiões, entre 1930 e 1932. Nestas viagens, foi recebido em 66 hotéis, parques de campismo e caravanismo e em outros alojamentos e foi atendido em 184 restaurantes e cafés (ou seja, um total de 250 estabelecimentos). Apenas no caso de um estabelecimento o atendimento foi recusado, devido à origem étnica do casal.

Decorrido um período de seis meses, o autor aplicou um questionário a esses mesmos estabelecimentos. Nesse questionário, era perguntado se receberiam e 
atenderiam clientes de origem chinesa. Do total de 128 respostas, 92\% responderam "Não" e a quase totalidade dos restantes respondeu "Depende das circunstâncias". Apenas um estabelecimento, dos 128 inquiridos, respondeu "Sim" (LAPIERE, 1934, p. 234).

Esses dados indicam que a verbalização predominante, de acordo com a qual o acesso aos clientes chineses é negado, não é materializada na esmagadora maioria dos casos, embora, a princípio, fizesse parte do imaginário manifesto pelos agentes. LaPiere (1934, p. 237) conclui, relativamente a esta questão, que produzir "um questionário é barato, fácil e mecânico", enquanto "o estudo do comportamento humano é demorado" e "intelectualmente fatigante".

Para caracterizar o comportamento efetivo dos indivíduos não basta, desta forma, colocar um conjunto de perguntas, tornando-se necessário observar as práticas ou, pelo menos, combinar diversas técnicas de recolha de informação. Caso contrário, a relação entre atitudes sociais e padrões comportamentais corre o risco de ser ignorada, na análise da realidade social.

Já numa negação à lógica binária de termos como "consistência" e "inconsistência" frente às práticas e representações, Kendler e Kendler (1949) buscaram explicações para fenómenos similares ao encontrado por LaPiere. Os agentes sociais poderiam realizar ações desassociadas das atitudes, se expostos a cenários antagónicos. Nesse caso hipotético, a necessidade humana de aceitação moldaria a forma de estar no mundo.

Como pontuam Bourdieu (2008) e Ander-Egg (1978) sobre as técnicas de inquirição, Kendler e Kendler (1949) também falam da interferência do investigador sobre o resultado do estudo. O pesquisador é uma força a levar-se em conta no momento de recolha de dados, uma "variável contribuinte na medição dos fenómenos" (KENDLER; KENDLER, 1949, p. 27). Já na avaliação de consistência e inconsistência dos comportamentos, é preciso considerar que os investigadores estão a fazer um julgamento das respostas aferidas pela inquirição, e não sobre uma observação direta de práticas. Kendler e Kendler (1949) dão como exemplo o caso de um indivíduo que afirma gostar de filmes, mas que vai ao cinema com pouca frequência. Perante esses dados, 0 investigador poderia acreditar existir uma "inconsistência". Entretanto, se por exemplo uma variável adicional for integrada na análise, a interpretação pode ser alterada de forma significativa: o inquirido vê apenas filmes que têm boa avaliação dos críticos. Como notam Kendler e Kendler (1949, p. 28), a questão empírica não consiste em saber porque o comportamento é inconsistente, mas em saber quais as variáveis que determinam as respostas.

Num diálogo com a sociologia do conhecimento, seria o mesmo que problematizar o mecanismo de elaboração social da realidade (BERGER; LUCKMANN, 2004). Ou seja, qual a influência que as representações exercem sobre os pressupostos que as ciências sociais - em especial os estudos da comunicação - têm das atividades quotidianas. Quando os utilizadores da Internet afirmam passar um determinado número de horas online por dia, os questionários ou as entrevistas estão a medir uma prática (ato concreto de utilização) ou uma representação (simulacro da utilização)?

As técnicas de recolha de informação assentes na inquirição ainda constituem recursos importantes para a análise da realidade (BRYMAN, 2012; BORDENS; ABBOTT, 2014). Disso não há dúvidas. Porém, parecem também assimilar distorções (LAPIERE, 1934; ROBERTS, 1985), ainda mapeadas com limitações, quando o fenómeno estudado 
é o impacto das TIC no tecido social (MONTARGIL; MIRANDA; DI FÁTIMA, 2019).

A própria limitação da memória humana de curto e de longo prazo, em entrevistas ou questionários, é uma força modeladora entre o que as pessoas afirmam fazer e o que fazem de facto. Nesse caminho, Roberts (1985) fala da recordação seletiva dos acontecimentos diários. Logo, faria sentido questionar se métodos de inquirição estão a medir as lembranças - reféns da seletividade - ou eventos quotidianos - assentes em práticas concretas. Quando um utilizador da Internet se depara com perguntas sobre temas com elevada probabilidade de reprovação social como, por exemplo, o consumo de pornografia ou o pagamento de direitos de autor no consumo de música e de filmes, as técnicas de inquirição estarão a medir práticas ou representações? Na literatura, "está bem estabelecido que falsas memórias de eventos podem ser tão vivas quanto memórias reais" (BORDENS; ABBOTT, 2014, p. 344).

Noutra linha de argumentação, Foddy (1996) salienta que quanto mais distante o acontecimento, maior é a probabilidade das respostas não retratarem a realidade. "Seria bom ter respostas precisas à pergunta do número de vezes que os entrevistados foram ao cinema nos últimos 12 meses, mas é altamente improvável que a maioria deles se lembre, com precisão, num período tão longo" (BRYMAN, 2012, p. 258). Essas reflexões parecem ainda mais relevantes para as sociedades altamente dinâmicas, marcadas pela produção, circulação e consumo de fluxos informativos em escala sem precedentes na história humana (MARTINIR, 2017; CASTELLS, 2013; CARDOSO, 2007).

Objeto sensível na sociedade de informação, a privacidade é levada à prova ao mesmo ritmo de popularização das TIC. Nesse ponto, para muitos estudos, os utilizadores da Internet podem estar a criar uma dissociação entre o discurso e as práticas online (LAl; LIANG; HUI, 2018; NORBERG; HORNE; HORNE, 2007). "As evidências empíricas e anedóticas mostram que os indivíduos estão dispostos a trocar suas informações e dados pessoais por recompensas relativamente pequenas" (KOKOLAKIS, 2017, p. 122). Por um lado, afirmam nas entrevistas e questionários estar muito preocupados com a privacidade e segurança na rede (OBERCOM, 2010; HFS RESEARCH, 2016). Por outro, têm práticas online que flexibilizam o acesso aos seus dados (KOKOLAKIS, 2017; CARRASCAL el al., 2013; BROWN, 2001).

Uma investigação conduzida por Dienlin e Trepte (2015), por exemplo, descobriu que a preocupação com a privacidade não parece estar diretamente correlacionada com os hábitos online. Indivíduos que se dizem especialmente preocupados com a privacidade na Internet são os mesmos que disponibilizam dados pessoais em sites como Facebook ou Twitter, como fotos familiares, número de telefone móvel e religião professada. Em alguns casos, a decisão de flexibilizar a privacidade parece assentar num processo de decisão racional, em que são avaliados custos e benefícios de cada opção. "Os utilizadores de Internet preferem trocar as informações por recompensas monetárias ou serviços aprimorados mais que trocá-los por serviços gratuitos adicionais e anúncios direcionados" (CARRASCAL el al., 2013, p. 189).

Brown (2001) também identificou padrões similares fora da Internet, no mundo offline. Numa investigação sobre fidelização dos clientes aos supermercados, o autor descobriu que as reclamações quanto à privacidade não impediam que os consumidores aceitassem fazer um cartão de desconto nas compras, mesmo que tivessem que partilhar seus dados pessoais. "Isso talvez apresente um paradoxo. Enquanto nossos inquiridos expressavam preocupações gerais com a privacidade, eles também estavam dispostos a 
trocá-la por ganhos muito pequenos" (BROWN, 2001, p. 18).

As técnicas de recolha de informação empírica baseadas na inquirição constituem, ainda, lentes importantes para a observação e caracterização da realidade, mas também revelam distorções entre as práticas e as representações (LAPIERE, 1934; FODDY, 1996). Dos estudos políticos (ANDERSON; SILVER, 1986) às investigações no campo da educação (HAGBURG, 1968), a validação dos resultados constitui um desafio para o investigador. A questão é particularmente relevante do ponto de vista científico e do potencial de valorização para diferentes stakeholders, como os órgãos de imprensa, as agências de publicidade ou as instituições públicas.

Se a Internet é um espaço de comunicação, informação e entretenimento, mapear os comportamentos online permite entender como as sociedades estão a ser edificadas. Desenvolver abordagens para caracterizar e estudar os comportamentos online consiste, deste modo, num desafio premente, sobretudo numa época que as TIC integram, em alguns contextos, a vida da maioria da população.

\section{Nota metodológica}

O Living Lab on Media Content and Platforms (LLMCP) é um projeto de investigação liderado pela Escola Superior de Comunicação Social (ESCS), do Instituto Politécnico de Lisboa (IPL), em consórcio com a Universidade de Aveiro, o Instituto Politécnico de Leiria, o Instituto Politécnico de Santarém e a Innovation Makers, empresa privada do setor tecnológico. Financiado pela Fundação para a Ciência e a Tecnologia (FCT) desde 22 de março de 2018, o objetivo do projeto consiste em estudar os hábitos de utilização da Internet, através de um painel online de utilizadores baseado na região de Lisboa.

Desenvolver um painel não é uma ideia original. A Marktest e a Nielsen, por exemplo, têm vindo a apostar em iniciativas semelhantes (MONTARGIL; MIRANDA; DI FÁTIMA, 2019). Porém, essa é a primeira vez que um centro público de ensino em Portugal e, provavelmente, na União Europeia, avança com uma pesquisa dessa natureza. Como era de se esperar, elaborar um painel apresenta dilemas e desafios de múltiplas dimensões que, pelo pioneirismo do LLMCP, merecem ser examinados.

Este estudo de caso segue as propostas de Yin (2001) quanto ao 'modelo exploratório', com enfoque incorporado: dilemas tecnológicos e desafios participativos. A recolha de dados teve por base a observação direta do painel, a leitura do projeto de investigação e o exame dos seus sistemas de informação, mas também o acesso privilegiado aos membros do LLMCP. O objetivo consiste em analisar os bastidores do trabalho da equipa, a partir do passo-a-passo da criação do painel, ao longo de 2018 . 0 estudo de caso justifica-se pelo pioneirismo do projeto, ainda numa etapa inicial, mas que procura a possibilidade de reprodução de métodos e abordagens e de verificação das conclusões (MORSE, 2010). 


\section{Motivações e barreiras à participação}

Durante o segundo semestre de 2018, o LLMCP desenvolveu uma extensão do Google Chrome capaz de monitorizar, em tempo real, as tendências de navegação num dos browsers mais populares entre os portugueses (STATCOUNTER, 2018). Para chegar ao ponto de arranque, a equipa de investigação executou um conjunto de procedimentos metodológicos, teóricos e operacionais na elaboração tanto da ferramenta de monitorização quanto na promoção do painel e captação de utilizadores da Internet.

Num primeiro momento do projeto, a equipa de investigação procurou experiências semelhantes e referências que pudessem apoiar as decisões do LLMCP. Entre as temáticas exploradas contava-se a legislação sobre a proteção dos dados pessoais, a privacidade online, a investigação sobre a relação entre práticas e representações nas ciências sociais e humanas e a análise sobre as limitações das técnicas de inquirição.

Nas reuniões de equipa foram consideradas e discutidas, inicialmente em registo de brainstorming, várias alternativas para a monitorização do comportamento online dos participantes num estudo de painel. Entre as alternativas consideradas, com maior ou menor grau de maturidade na discussão, incluem-se (i) o desenvolvimento de um sistema que assegurasse a passagem do tráfego de navegação entre o computador do utilizador e o servidor do Internet Service Provider por um servidor intermédio (servidor proxy), permitindo assim o registo da navegação, (ii) a utilização de tecnologia de "filmagem" do ecrã do computador do utilizador, eventualmente combinada com tecnologia eye tracking, (iii) a utilização de um browser próprio, a desenvolver pela equipa e (iv) a utilização de browsers existentes no mercado, adaptados de forma a permitir o registo de informação das sessões de navegação.

Na sequência de várias reuniões de discussão e avaliação de alternativas, durante alguns meses, a escolha recaiu no desenvolvimento de uma extensão para Google Chrome que permitisse recolher a informação registada pelo browser no histórico de navegação. Embora a opção apresentasse algumas limitações, permitindo por exemplo apenas a monitorização de um browser, era também a solução com a melhor relação custo-benefício, a menos invasiva para os membros do painel e com tempo de desenvolvimento mais adequado à calendarização global do projeto. Esta alternativa foi testada através de uma prova de conceito, que permitiu o teste dos pressupostos centrais da solução, antes do desenvolvimento da aplicação final.

O desenvolvimento da extensão levou cerca de dois meses, utilizados essencialmente na elaboração da base de dados, na programação do mecanismo de recolha do histórico de navegação e na criação da interface gráfica.

A partir de testes unitários e integrados, procedeu-se à identificação de erros de operação da aplicação e à validação do processo de recolha de informação. Ou seja, procurou-se que os dados recolhidos na monitorização refletissem da melhor forma possível o histórico de navegação do utilizador. Em paralelo, o projeto criou um formulário de registo (Figura 1) com apenas oito perguntas, que combinam a caracterização sociodemográfica (idade, género, estado civil...) e a utilização da Internet (dispositivos, utilizadores.... $)^{1}$. 


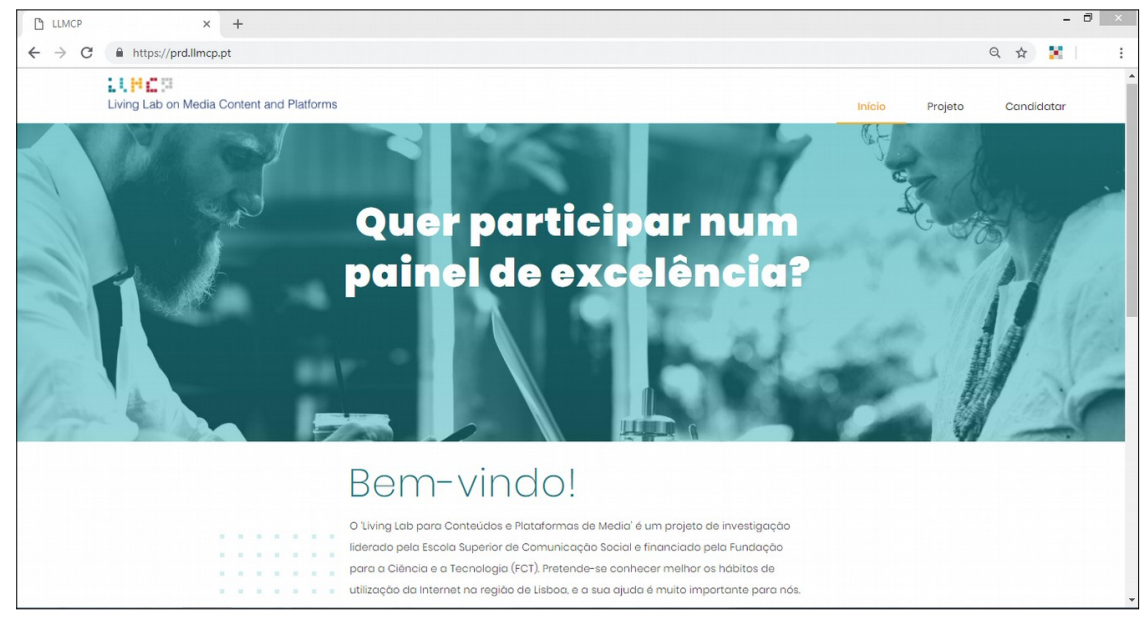

Figura 1: Homepage do formulário de registo. Fonte: LLMCP.

A extensão e o formulário foram planeados e concebidos de forma a preservar o anonimato e a confidencialidade dos utilizadores e da informação recolhida, segundo o Regulamento Geral de Proteção de Dados (RGPD), em vigor desde 25 de maio de 2018, na União Europeia (EU). Uma das funcionalidades implementadas na extensão, por exemplo, permite que o utilizador suspenda de forma imediata e simples a monitorização, com um único comando e sem a necessidade de qualquer intervenção do LLMCP (Figura 2).

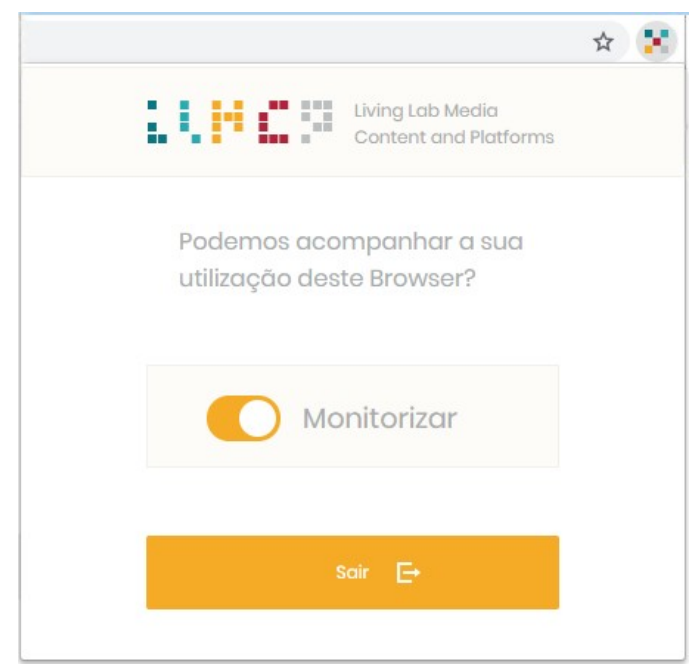

Figura 2: Extensão do Google Chrome. Fonte: LLMCP.

Para garantir o controlo da amostra, fundamental nas ciências sociais (ANDEREGG, 1978), o registo funciona como um formulário de candidatura ao painel, gerida com ajuda de um backoffice (Figura 3). Uma das características do backoffice é a ficha de participante, que permite a eventual segmentação da amostra. 


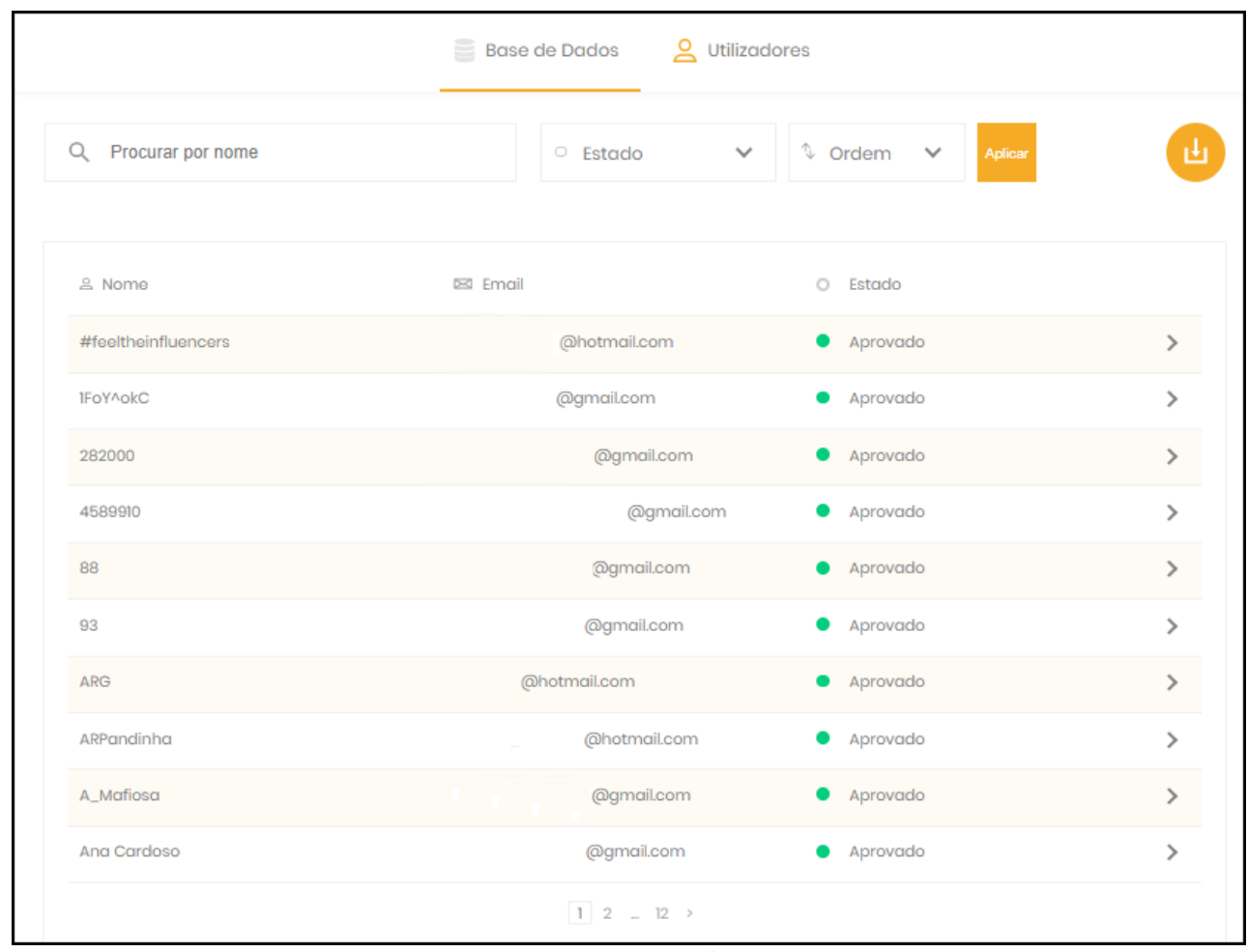

Figura 3: Backoffice do formulário de candidatura.

Fonte: LLMCP.

O processo para integrar o painel encontra-se essencialmente organizado em três etapas, inter-relacionadas. Na primeira, o utilizador precisa aceder ao formulário e submeter a sua candidatura. Optou-se por pedir um nickname do candidato, e não necessariamente o nome real, de forma a minimizar a possibilidade de identificação do utilizador. Na segunda etapa, o LLMCP avalia o pedido com base nos dados fornecidos, e pode aceitar ou rejeitar a candidatura. Na terceira, se aprovado, o candidato recebe um email com instruções para fazer o download, instalar e ativar a extensão no Google Chrome.

O minucioso processo para integrar o painel apresentou vantagens e limitações que, de certa forma, tendem a condicionar os resultados deste estudo. Por um lado, garantiu um rígido controlo da amostra, com possibilidade de identificar o perfil sociodemográfico e tecnológico dos membros do painel. Por outro, fez com que um número significativo de candidatos, cerca de 45,5\% ( $n=51)$, não ultrapassasse pelo menos uma das fases de registo. Entre os problemas mais recorrentes estão, por exemplo, os candidatos aprovados que acabaram por não concluir a instalação ou ativação da extensão no Chrome. É plausível pensar que, em alguns casos, o e-mail oficial do LLMCP, com a comunicação da aprovação e as instruções de instalação da aplicação, tenha sido considerado pelo serviço de e-mail do candidato como spam (situação confirmada em alguns testes, realizados pela equipa).

Enquanto os 'dilemas tecnológicos' deixavam de existir, com as componentes técnicas em pleno funcionamento, surgiam os 'desafios participativos'. Em resumo, como mobilizar as candidaturas para o painel? Na primeira etapa, ainda de teste, o LLMCP definiu que os alunos, funcionários e docentes da Escola Superior de Comunicação Social 
(ESCS) eram o público-alvo.

A equipa de investigação elaborou um plano de comunicação ancorado em três ações promocionais. Primeira, fez a apresentação do projeto em sala de aula para 40 turmas da Escola, chegando a quase 1.500 alunos. Segunda, divulgou conteúdos informativos no site e nas redes sociais (Facebook, Twitter, Instagram) da instituição. Terceira, adotou a estratégia de abordagem individual dos alunos em ambiente informal, em saídas de campo periódicas, com explicações mais detalhadas da iniciativa.

Em dois meses, o LLMCP conseguiu alcançar a cota mínima de participantes da ESCS: 112 membros registados e 61 a monitorizar com regularidade. Como é de supor, a amostra da primeira fase reflete o cenário sociodemográfico da Escola. O painel é formado por $73,2 \%$ de mulheres e $26,8 \%$ de homens. Dos utilizadores, $50,0 \%$ têm menos de 21 anos, seguidos por indivíduos entre 22 e 24 anos (25,9\%) e entre 25 e 29 anos $(14,3 \%)$. Com a menor coorte estão os docentes e os funcionários da ESCS, todos acima dos 30 anos $(9,8 \%)$. Indivíduos que declaram apenas estudar ocupam a maior fasquia do painel $(76,4 \%)$, seguidos pelos que dizem estudar e trabalhar $(18,2 \%)$, apenas trabalhar $(4,5 \%)$ e estar desempregados $(0,9 \%)$. Os dados sociodemográficos são confirmados pelo próprio estado civil maioritário da amostra, formado sobretudo por jovens do ensino superior: $93,7 \%$ são solteiros, enquanto $6,3 \%$ são casados ou vivem em união de fato.

Durante as abordagens individuais aos estudantes, previstas no plano de comunicação, a equipa do LLMCP apurou as motivações e os impedimentos à participação no projeto. As perguntas colocadas foram: por que aceita ou não aceita participar no painel? A principal motivação de $58 \%$ dos inquiridos é a 'utilidade dos dados' da monitorização para o universo académico, empresas e pesquisas de consumo em geral, seguida por 'receber o incentivo' - um power bank por utilizador -, 'ajudar o projeto' da ESCS e 'não sabem'. Em larga medida, os dados do Gráfico 1 parecem refletir a perceção que um grupo de alunos $(n=33)$, todos de algum curso de Comunicação (Jornalismo, Relações Públicas, Publicidade e Marketing, etc), tem da forma como empresas, órgãos públicos ou produtores de conteúdo poderiam empregar as informações sobre os comportamentos online no melhoramento de produtos e de serviços. Descobertas similares levaram Carrascal et al. (2013) a argumentar que os utilizadores de Internet estão mais dispostos a trocar os dados pessoais por incentivos monetários e melhoria de serviços, por exemplo, do que por publicidade dirigida.

Gráfico 1: Por que ACEITA participar no painel? $(n=33)$

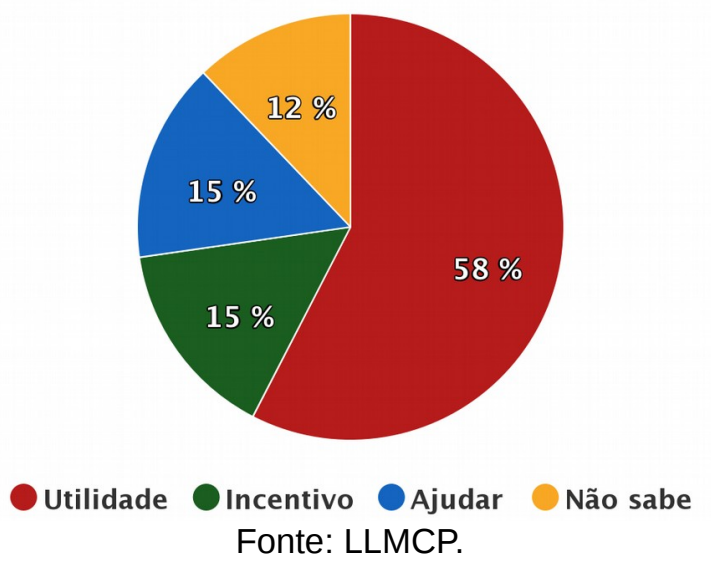


Por outro lado, 44\% dos indivíduos abordados não aceitaram participar no painel por causa da 'preocupação com a privacidade', mesmo quando explicados que todo o registo e a monitorização são anónimos e seguem o Regulamento Geral de Proteção de Dados (RGPD). Os alunos que 'não sabem' exatamente qual o impedimento representam $25 \%$ dos inquiridos, seguidos pelos que disseram ter 'problemas técnicos' no computador e 'não utilizar o Google Chrome' como navegador.

O Gráfico 2 parece indicar ao menos três importantes argumentos que podem guiar, numa lógica de reprodutibilidade científica (MORSE, 2010), o planeamento de iniciativas semelhantes, com base num grupo específico de estudantes $(n=48)$. Primeiro, a preocupação com a privacidade é a mais importante barreira à participação em projetos sobre os comportamentos online com base em monitorização. Depois, que tal constrangimento pode ser ocultado por respostas evasivas, como "não sei", "nunca pensei nisso" e "participo noutro momento", interferindo, assim, nos resultados apurados. Por último, que a própria escolha das tecnologias para recolha de dados pode constituir-se como um interdito. No caso do LLMCP, a opção pelo Chrome parece acertada. Só uma parcela muito pequena dos que se recusaram a integrar o painel, por exemplo, o fizeram por não utilizar esse browser da Google (10\%).

Gráfico 2: Por que NÃO ACEITA participar no painel? $(n=48)$.

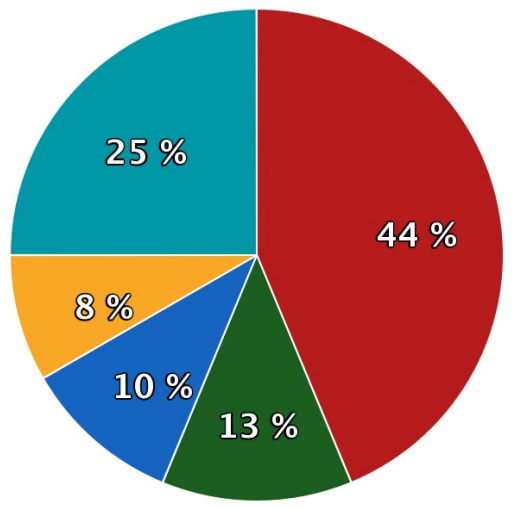

Privacidade Técnico Chrome Outro Não sabe Fonte: LLMCP.

\section{Conclusão}

Este artigo analisou os desafios e as premissas para a construção de um painel online de utilizadores de Internet, com foco na experiência pioneira do Living Lab on Media Content and Platforms (LLMCP). A partir do trabalho da equipa, procurou-se examinar os bastidores do projeto ao longo de 2018, ano de lançamento da extensão do Chrome e execução do plano de comunicação. Além do estudo de caso exploratório (YIN, 2001), com enfoque incorporado: dilemas tecnológicos e desafios participativos, o artigo também problematizou o impacto dos métodos de inquirição nas pesquisas dos comportamentos online.

O cruzamento dos primeiros resultados do LLMCP revela que os investigadores 
vivem uma batalha entre a 'utilidade dos dados' e a 'invasão de privacidade'. Embora a amostra seja constituída por um grupo específico de utilizadores da Internet (estudantes do ensino superior), os trabalhos de outros autores ajudam a apontar que esse é um dilema transversal a diferentes realidades complexas (KOKOLAKIS, 2017; CARRASCAL el al., 2013). Estudos futuros no âmbito desse projeto poderiam testar, por exemplo, a persistência do paradoxo da privacidade (BROWN, 2001) nos jovens utilizadores da Internet. Ou, ainda, o impacto do paradoxo frente as práticas e representações (LAPIERE, 1934; FODDY, 1996).

A experiência do LLMCP também sugere três argumentos para os Estudos da Internet pelas ciências sociais e humanidades. Primeiro, que os métodos mais tradicionais precisam ser questionados numa sociedade hiperconectada, com profundas transformações de todas as suas estruturas e vidas em rede. Segundo, que a popularização das TIC é uma oportunidade para a criação de métodos inovadores, com base na observação direta dos comportamentos online e, no rescaldo, offline. Terceiro, que essas abordagens - como um painel online de utilizadores da Internet - precisam desenvolver estratégias para vencer o medo da vigilância e do controlo que paira nas sociedades de informação e que pode limitar as investigações mais robustas das novas formas de estar no mundo.

\section{Agradecimentos}

O Living Lab on Media Content and Platforms (LLMCP) é financiado pela Fundação para a Ciência e a Tecnologia (FCT), com apoio da União Europeia. Referência: LISBOA01-0145-FEDER-023937.

\section{Referências}

ADAPA, S. Global e-banking trends: evolution, challenges and opportunities. In: SARLAK, M. A.; HASTIANI, A. A. (Org.). E-banking and emerging multidisciplinary processes: social, economical and organizational models. Hershey, PA: Business Science Reference, 2011, p. 1-16.

ANDER-EGG, E. Introducción a las técnicas de investigación social. Buenos Aires: Humanitas, 1978.

ANDERSON, B. A.; SILVER, B. D. Measurement and mismeasurement of the validity of the self-reported vote. American Journal of Political Science, Bloomington, v. 30, n. 4, p. 771-785, 1986.

BERGER, P.; LUCKMANN, T. A construção social da realidade. Rio de Janeiro: Vozes, $24^{\mathrm{a}}$ Ed., 2004.

BORDENS, K. S.; ABBOTT, B. B. Research and design methods: a process approach. Nova York: McGraw-Hill, 9a Ed., 2014. 
BOURDIEU, P. A miséria do mundo. Petrópolis: Vozes, 2008.

BROWN, B. Studying the Internet experience. Bristol, Hewlett-Packard Company e HP Laboratories, 2001.

BRYMAN, A. Social research methods. Oxford: Oxford University Press, 4ª Ed., 2012.

CARDOSO, G. A mídia na sociedade em rede. Rio de Janeiro: FGV, 2007.

CARDOSO, G., LAPA, T.; DI FÁTIMA, B. People are the message? Social mobilization and social media in Brazil. International Journal of Communication, Los Angeles, v. 10, p. 3909-3930, 2016.

CARRASCAL, J. P. et al. Your browsing behavior for a Big Mac: economics of personal information online. In: Atas da 22nd International Conference on World Wide Web, Rio de Janeiro: World Wide Web Consortium (W3C), 2013, p. 189-200.

CASTELLS, M. A sociedade em rede. A era da informação: economia, sociedade e cultura. Lisboa: Fundação Calouste Gulbenkian, 2002.

CASTELLS, M. O poder da comunicação. Lisboa: Fundação Calouste Gulbenkian, 2013.

DIENLIN, T.; TREPTE, S. Is the privacy paradox a relic of the past? An in-depth analysis of privacy attitudes and privacy behaviours. European Journal of Social Psychology, Hoboken, v. 45, n. 3, p. 285-297, 2015.

ESPANHA, R. Informação sobre saúde: as fontes de informação e a construção da autonomia. Factores de Risco, Lisboa, n. 40, p. 15-23, 2016.

FODDY, W. Como perguntar: teoria e prática da construção de perguntas em entrevistas e questionários. Lisboa: Celta, 1996.

GREEN, L. The Internet: an introduction to new media. Oxford: Berg Publishers, 2010.

HAGBURG, E. C. Validity of questionnaire data: reported and observed attendance in an adult education program. The Public Opinion Quarterly, Oxford, v. 32, n. 3, p. 453-456, 1968.

HFS RESEARCH. The state of cybersecurity and digital trust: identifying cybersecurity gaps to rethink state of the art. Cambridge: The Services Research Company ${ }^{\mathrm{TM}}, 2016$.

HUTCHINS, B.; ROWE, D. Sport beyond television: the Internet, digital media and the rise of networked media sport. Londres: Routledge, 2012.

IBOPE CONECTA. 95\% dos internautas brasileiros assistem TV enquanto usam Internet. São Paulo: Ibope, 2018. 
INE. Sociedade da Informação e do Conhecimento: inquérito à utilização de Tecnologias da Informação e da Comunicação pelas famílias. Lisboa: Instituto Nacional de Estatística, 2017.

KENDLER, H. H.; KENDLER, T. S. A methodological analysis of the research area of inconsistent behavior. Journal of Social Issues, Hoboken, v. 5, n. 3, p. 27-31, 1949.

KOKOLAKIS, S. Privacy attitudes and privacy behaviour: a review of current research on the privacy paradox phenomenon. Computers \& Security, Amsterdam, v. 64, p. 122-134, 2017.

LAI, C. Y.; LIANG, T. P.; HUI, K. L. Information privacy paradox: A neural science study, In: Atas da 22nd Pacific Asia Conference on Information Systems, Yokohama: Association for Information Systems (AIS), 2018. Disponível em: https://aisel.aisnet.org/pacis2018/247. Acesso em: 19 fev. 2018.

LAPIERE, R. T. Attitudes vs. Actions. Social Forces, Oxford, v. 13, n. 2, p. 230-237, 1934.

MARKTEST. Barómetro de telecomunicações - voz móvel. Lisboa: Grupo Marktest, 2018.

MARKTEST. Um dia das nossas vidas na Internet: estudo de hábitos digitais dos portugueses. Lisboa: Nova Expressão, 2015.

MARTINIR, R. Sociedade da informação: para onde vamos. São Paulo, Trevisan, 2017.

MONTARGIL, F.; MIRANDA, S.; DI FÁTIMA, B. Práticas e representações: desafios da monitorização dos comportamentos online. In: VI Congresso Internacional de Comunicação, Jornalismo e Espaço Público, Sessão 3, Coimbra, Universidade de Coimbra, 2019.

MORSE, J. Procedures and practice of mixed method design: maintaining, control, rigor and complexity, In: Tashakkori, A.; Teddlie, C. (Org.). Sage handbook of mixed methods in social \& behavioral research. Los Angeles: Sage, p. 339-352, 2010.

NORBERG, P. A.; HORNE, D. R.; HORNE, D. A. The privacy paradox: personal information disclosure intentions versus behaviours. The Journal of Consumer Affairs, Hoboken, v. 41, n. 1, 2007, p. 100-126.

OBERCOM. A Internet em Portugal: sociedade em rede 2014. Lisboa: Observatório da Comunicação, 2014.

OBERCOM. A utilização de Internet em Portugal. Lisboa: Observatório da Comunicação, 2010.

PEW CENTER. Social media update 2016. Internet \& Technology, 2016. Disponível em: www.pewinternet.org/2016/11/11/social-media-update-2016/. Acesso em: 19 fev. 2018. 
ROBERTS, J. V. The attitude-memory relationship after 40 years: a meta-analysis of the literature. Basic and Applied Social Psychology, Abingdon-on-Thames, v. 6, n. 3, p. 221241, 1985.

SALAVERRÍA, R. Ciberperiodismo en Iberoamérica: marco general. In: SALAVERRÍA, R. (Org.). Ciberperiodismo en Iberoamérica. Barcelona: Ariel, p. 16-35, 2016.

STATCOUNTER. Top desktop, tablet \& console browsers per country. Nova Yorque: Global Stats Tool, 2018.

WEST, D. M. Digital divide: improving Internet access in the developing world through affordable services and diverse content. Center for Technology Innovation at Brookings, Washington, Ed. February, p. 1-30, 2015.

YIN, R. K. Estudo de caso: planejamento e métodos. Porto Alegre: Bookman, 2001.

Recebido em dia 08 de fevereiro de 2019. Aprovado em dia 08 de março de 2019. 